\title{
Servicios Ecosistémicos en Latinoamérica. De la investigación a la acción
}

\author{
Pedro Laterra'; Berta Martín-López; Matías Mastrángelo ${ }^{3}$ \& Lucas A. Garibaldi ${ }^{4}$ \\ 1,3 Programa Sistemas Socio-Ecológicos, Dpto. de Análisis de Sistemas Complejos, Fundación Bariloche - Consejo Nacional de \\ Investigaciones Científicas y Técnicas (CONICET). San Carlos de Bariloche, Río Negro, Argentina. ${ }^{2}$ Leuphana University of \\ Lüneburg, Faculty of Sustainability, Institute of Ethics and Transdisciplinary Sustainability Research. Lüneburg, Germany. \\ ${ }^{3}$ Grupo de Agroecosistemas y Paisajes Rurales (GEAP), Unidad Integrada Balcarce: INTA Balcarce y Facultad de Ciencias \\ Agrarias, UNMDP. Balcarce, Buenos Aires, Argentina. ${ }^{4}$ Instituto de Investigaciones en Recursos Naturales, Agroecología y \\ Desarrollo Rural (IRNAD), Sede Andina, Universidad Nacional de Río Negro (UNRN) y Consejo Nacional de Investigaciones
}

RESEÑA: Las investigaciones en servicios ecosistémicos (SE), impulsadas por la potencialidad de este enfoque para orientar la toma de decisiones a favor de territorios más sustentables y con mayor justicia socioambiental, representan en la actualidad uno de los campos más activos de la ecología (Abson et al. 2014; Hevia et al. 2017; Seppelt et al. 2011). La importancia de los SE para el bienestar humano y la necesidad de detener la pérdida alarmante de la biodiversidad que los sostienen han convocado grandes esfuerzos internacionales para su difusión, como, por ejemplo, la Evaluación de los Ecosistemas del Milenio (MEA 2005) o, más recientemente, la Plataforma Intergubernamental de Biodiversidad y Servicios Ecosistémicos (IPBES) (Díaz et al. 2015). Sin embargo, el ritmo con que los SE son internalizados en la toma de decisiones públicas y privadas aún se encuentra muy alejado de lo esperado a partir de esos esfuerzos (Laurans et al. 2013; Laurans and Mermet 2014; Martinez-Harms et al. 2015).

Los marcos conceptuales que integran los SE a las decisiones sobre el uso de la tierra se distinguen de los enfoques previos por su capacidad de incorporar al análisis la existencia de múltiples actores sociales con distintas demandas, distintas visiones del mundo y, en definitiva, con relaciones de poder asimétricas (Bennett et al. 2015; BerbésBlázquez et al. 2016; Díaz et al. 2015; Pascual et al. 2017). En ese marco, la capacidad de los ecólogos para lograr una mayor incidencia del enfoque de SE en la toma de decisiones exige necesariamente una progresiva integración con otras disciplinas, así como con los saberes, la percepción, las preferencias, la legitimación y el compromiso de otros actores sociales, entre los que se incluyen afectadores (propietarios), afectados (propietarios y no propietarios) y tomadores de decisiones públicas (legisladores y funcionarios) (Albert et al. 2014; Cowling et al. 2008; Daily et al. 2009; Posner et al. 2016). La necesidad de esa integración inter y transdisciplinaria también ha sido puesta de manifiesto para nuestra propia región (Balvanera et al. 2012; Cáceres et al. 2016; Mastrangelo et al. 2015).

En este número especial de Ecología Austral se ofrece un pequeño recorrido por el enfoque de SE a través de la mirada de investigadores latinoamericanos, sobre la base de 7 de los 205 trabajos presentados en el Cuarto Congreso Internacional de Servicios Ecosistémicos en los Neotrópicos (CISEN4), realizado en Mar del Plata, Argentina, en octubre 2015 (disponibles en CISEN4 2015). Aunque se trata de una muestra pequeña, su lectura pone en evidencia los logros y las dificultades propias de distintas etapas que bajo este enfoque intentan vincular el bienestar humano con la promoción de la biodiversidad, así como las características propias e idiosincráticas de la aproximación de SE en Latinoamérica.

Entre los retos a los que se enfrenta el enfoque de SE se destacan: a) la comprensión profunda y holista de las contribuciones más intangibles al bienestar humano (i.e., servicios culturales [Chan et al. 2012, 2016; van Riper et al. 2017]), b) la representación espacial del suministro y consumo de SE por parte de diferentes actores sociales con el fin de informar políticas de manejo y planificación del territorio (Crossman et al. 2013; Laterra and Nahuelhual 2015; Martínez-Harms and Balvanera 2012; Nahuelhual et al. 2015; Tallis and Polasky 2009; Viglizzo et al. 2012), c) el análisis de los sistemas de gobernanza que favorecen la internalización del enfoque de SE a las políticas públicas, la gestión sustentable de los SE y la distribución justa y equitativa de sus beneficios entre diferentes actores sociales (Bennett et al. 2015; Daw et al. 2011; Fisher et 
al. 2014), y d) las prácticas y tecnologías de manejo para promover la biodiversidad y los SE (Dale and Polasky 2007; Garibaldi et al. 2017; Kremen and Miles 2012).

Con respecto al primer reto (i.e., comprensión holista e integradora de los SE al bienestar humano), Bentacourt y Nahuelhual (este número) desafían la taxonomía de los SE al mostrarnos, desde una perspectiva sociológica, que las plantas medicinales utilizadas por los mapuches de Panguipulli (Chile) aportan al bienestar de manera integrada y satisfacen tanto necesidades vinculadas a la salud como al afecto, el ocio y la libertad. Los autores remarcan el encuentro entre la perspectiva de SE y la cosmovisión mapuche a través de las palabras de una "machi" "... Hoy en día hay hartas gentes enfermas, pero no sólo porque no se cuiden, sino porque tampoco se preocupan de la naturaleza y nosotros somos naturaleza y ya no quedan personas que sepan cuidarla. Los espiritus, los Ñgem, como decimos nosotros, muchos han muerto". En este sentido, Bentacourt y Nahuelhual, además, ponen de manifiesto la importancia del conocimiento local e indígena para llevar a cabo evaluaciones holistas de los SE, tal y como se está impulsando desde IPBES (e.g., Díaz et al. 2015; Tengö et al. 2014).

Con respectoal segundoreto, desde temprano se ha advertido que la capacidad para mapear SE es un requisito fundamental para identificar prioridades de manejo, y es el origen de una de las líneas de trabajo más importantes dentro de este paradigma. El trabajo de Teruya et al. (2017) ilustra la utilidad potencial de este tipo de estudios para identificar las áreas más valiosas como generadoras de SE cuando el territorio y la calidad de vida de sus habitantes es amenazado por cambios acelerados en el uso de la tierra, como los que ocurren en la cuenca del Arroyo Grande (Tunuyán, Mendoza). También en esta línea de investigación, el trabajo de Olaya Rodríguez et al. (2017) aporta características muy poco frecuentes en la bibliografía sobre evaluación y mapeo de SE, tanto por el tipo de SE abordado (la provisión de peces para la pesca continental en humedales interiores de Colombia) como por incorporar al análisis no solo las áreas de generación del SE sino también su propagación hasta las áreas de consumo. Al combinar los patrones de generación, propagación y demanda por beneficiarios directos e indirectos, Olaya Rodríguez et al. (2017) cuestionan la sustentabilidad de las actuales políticas de manejo del recurso pesquero y proveen criterios para su mejor gestión y conservación.

El análisis de la internalización del enfoque de SE a las políticas públicas y de la gestión sustentable de los SE, el tercer reto reconocido más arriba, ha sido el foco de cuatro de los trabajos seleccionados. En el primero de ellos, Cabrol y Cáceres (2017) se ocupan de la primera ley nacional que incorpora el concepto de SE como criterio para el ordenamiento territorial de bosques nativos y para orientar un esquema de compensaciones económicas ("Ley de Bosques Nativos"), con atención particular a su implementación en la provincia de Córdoba (Argentina). Aunque fue muy celebrada por la comunidad ambientalista, bajo el análisis de Cabrol y Cáceres, la implementación de esta ley muestra aristas que cuestionan su eficacia, tanto para proveer niveles mayores de conservación de la biodiversidad como para resolver conflictos claros entre los actores sociales que ostentan diferencias de poder y de vulnerabilidad.

Aguiar et al. (2017) se suman a un debate álgido sobre el pago por SE y sobre otros instrumentos económicos basados en el mercado de conservación, al caracterizar la eficacia de sus objetivos ambientales (incrementar la conservación) y sociales (disminuir la inequidad y la pobreza), así como sus relaciones con distintos factores institucionales. En contraste con estudios mayormente basados en consideraciones teóricas y ejemplos aislados, este trabajo se basa en información empírica de 60 proyectos situados en Latinoamérica. Al poner en relieve la incertidumbre actual en torno a los factores que controlan el éxito de estos instrumentos, los autores advierten sobre sus riesgos éticos, sociales y políticos.

A través de un estudio empírico sobre el uso silvopastoril de los bosques de ñire (Nothofagus antárctica), Rusch et al. (2017) reflexionan sobre la necesidad de gestión sustentable de paisajes más allá de la simple dicotomía entre conservar vs. no conservar. En este artículo se destacan las implicancias de alternativas de uso silvopastoril de bosques de ñire sobre beneficios públicos y privados en distintas escalas de tiempo. A fin de facilitar la incorporación de esa perspectiva temporal, los autores desarrollan y aplican un protocolo en el que se combinan modelos de la dinámica del ecosistema, la cuantificación de flujos de SE y un sistema de toma de decisiones. 
Finalmente, el cuarto trabajo dedicado a gobernanza e internalización de SE en las políticas públicas ofrece un ejemplo de la importancia de la legitimidad social como uno de los ingredientes fundamentales que caracterizan a los casos exitosos de incorporación de SE a la toma de decisiones (Posner et al. 2016). Desde esa perspectiva no parece casual que el caso de aplicación más exitoso seleccionado dentro de este número especial corresponda a la creación de un área protegida (humedales de la cuenca del Río Blanco, Mendoza, Argentina) a partir de la iniciativa y la participación colaborativa de los actores sociales en torno al diagnóstico general, a la identificación, a la evaluación y el mapeo de los SE, al diseño y a la formulación de la propuesta de creación (Rubio et al. 2017). Los autores de este trabajo ofrecen lecciones obtenidas a partir de esos procesos que merecen ser tomadas en cuenta en experiencias similares.

En relación al cuarto reto, Rey Benayas et al. (2017), a través de una síntesis de cuatro meta-análisis globales, demuestran que la restauración de humedales, agroecosistemas y bosques puede aumentar la biodiversidad y la provisión de SE de forma notable. Sin embargo, los autores advierten que las iniciativas por recuperar SE en base a técnicas de restauración deben tomar en cuenta la lentitud de estos procesos y que resulta preferible el mantenimiento de la integridad de los ecosistemas naturales.

A través de casos empíricos a escala local y de revisiones de literatura en Latinoamérica, los artículos aquí reunidos y comentados ofrecen nuevos elementos para encarar los retos de investigación y gestión formalizados recientemente en la comunidad científica de SE (Bennett et al. 2015). Esperamos así que este número especial contribuya tanto a la investigacióninterdisciplinary transdisciplinar de los SE, como a la aplicación de dicha aproximación en territorios de nuestra región con el fin de favorecer la sustentabilidad y la justicia socio-ambiental.

\section{REFERENCIAS}

Abson, D. J., H. von Wehrden, S. Baumgärtner, J. Fischer, J. Hanspach, W. Härdtle, H. Heinrichs, A. M. Klein, D. J. Lang, P. Martens, and D. Walmsley. 2014. Ecosystem services as a boundary object for sustainability. Ecol Econ 103: 29-37. doi:10.1016/j.ecolecon.2014.04.012.

Aguiar, S., G. Camba Sanz, and J. Paruelo. 2017. Instrumentos económicos basados en mercados para la conservación de la biodiversidad y los servicios ecosistémicos en Latinoamérica: ¿panacea o rueda cuadrada? Ecol Austral 27: 146-161.

Albert, C., J. Aronson, C. Fürst, P. Opdam. 2014. Integrating ecosystem services in landscape planning: requirements, approaches, and impacts. Springer.

Balvanera, P., M. Uriarte, L. Almeida-Leñero, A. Altesor, F. DeClerck, T. Gardner, J. Hall, A. Lara, P. Laterra, M. PeñaClaros, D. M. Silva Matos, A. L. Vogl, L. P. Romero-Duque, L. F. Arreola, Á. P. Caro-Borrero, F. Gallego, M. Jain, C. Little, R. de Oliveira Xavier, J. M. Paruelo, J. E. Peinado, L. Poorter, N. Ascarrunz, F. Correa, M. B. Cunha-Santino, A. P. Hernández-Sánchez, and M. Vallejos. 2012. Ecosystem services research in Latin America: The state of the art. Ecosyst Serv 2:56-70. doi:10.1016/j.ecoser.2012.09.006.

Bennett, E. M., W. Cramer, A. Begossi, G. Cundill, S. Díaz, B. N. Egoh, I. R. Geijzendorffer, C. B. Krug, S. Lavorel, E. Lazos, L. Lebel, B. Martín-López, P. Meyfroidt, H. A. Mooney, J. L. Nel, U. Pascual, K. Payet, N. P. Harguindeguy, G. D. Peterson, A. -H. Prieur-Richard, B. Reyers, P. Roebeling, R. Seppelt, M. Solan, P. Tschakert, T. Tscharntke, B. Turner, P. H. Verburg, E. F. Viglizzo, P. C. White, and G. Woodward. 2015. Linking biodiversity, ecosystem services, and human well-being: three challenges for designing research for sustainability. Curr Opin Environ Sustain, Open Issue 14:76-85. doi:10.1016/j.cosust.2015.03.007.

Bentacourt, R., L. Nahuelhual. 2017. Servicios ecosistémicos y bienestar local: caso de estudio sobre productos de medicina natural en Panguipulli, sur de Chile. Ecol Austral 27:099-112.

Berbés-Blázquez, M., J. A. González, and U. Pascual. 2016. Towards an ecosystem services approach that addresses social power relations. Curr Opin Environ Sustain 19:134-143. doi:10.1016/j.cosust.2016.02.003.

Cabrol, D., and D. Cáceres. 2017. Las disputas por los bienes comunes y su impacto en la apropiación de servicios ecosistémicos. La Ley de Protección de Bosques Nativos, en la Provincia de Córdoba, Argentina. Ecol Austral 27: 134-145.

Cáceres, D. M., F. Silvetti, and S. Díaz. 2016. The rocky path from policy-relevant science to policy implementation - a case study from the South American Chaco. Curr Opin Environ Sustain 19:57-66. doi:10.1016/j.cosust.2015.12.003.

Chan, K. M. A., P. Balvanera, K. Benessaiah, M. Chapman, S. Díaz, E. Gómez-Baggethun, R. Gould, N. Hannahs, K. Jax, S. Klain, G. W. Luck, B. Martín-López, B. Muraca, B. Norton, K. Ott, U. Pascual, T. Satterfield, M. Tadaki, J. Taggart, and N. Turner. 2016. Opinion: Why protect nature? Rethinking values and the environment. Proc Natl Acad Sci 113: 1462-1465. doi:10.1073/pnas.1525002113.

Chan, K. M. A., T. Satterfield, and J. Goldstein. 2012. Rethinking ecosystem services to better address and navigate cultural values. Ecol Econ 74:8-18. doi:10.1016/j.ecolecon.2011.11.011. 
CISEN4. 2015. Cuarto Congreso Internacional de Servicios Ecosistémicos en los Neotrópicos. GEAP. www.geap.com.ar/ cisen4/libro-resumenes/, Mar del Plata, Argentina.

Cowling, R. M., B. Egoh, A. T. Knight, P. J. O'Farrell, B. Reyers, M. Rouget, D. J. Roux, A. Welz, and A. WilhelmRechman. 2008. An operational model for mainstreaming ecosystem services for implementation. Proc Natl Acad Sci 105:9483-9488.

Crossman, N. D., B. Burkhard, S. Nedkov, L. Willemen, K. Petz, I. Palomo, E. G. Drakou, B. Martín-López, T. McPhearson, K. Boyanova, R. Alkemade, B. Egoh, M. B. Dunbar, and J. Maes. 2013. A blueprint for mapping and modelling ecosystem services. Ecosyst Serv. (special issue on mapping and modelling ecosystem services) 4:4-14. doi:10.1016/j.ecoser.2013.02.001.

Daily, G. C., S. Polasky, J. Goldstein, P. M. Kareiva, H. A. Mooney, L. Pejchar, T. H. Ricketts, J. Salzman, and R. Shallenberger. 2009. Ecosystem services in decision making: time to deliver. Front Ecol Environ 7:21-28.

Dale, V. H., and S. Polasky. 2007. Measures of the effects of agricultural practices on ecosystem services. Ecol Econ 64:286-296.

Daw, T., K. Brown, S. Rosendo, and R. Pomeroy. 2011. Applying the ecosystem services concept to poverty alleviation: the need to disaggregate human well-being. Environ Conserv 38:370-379.

Díaz, S., S. Demissew, J. Carabias, C. Joly, M. Lonsdale, N. Ash, A. Larigauderie, J. R. Adhikari, S. Arico, A. Baldi, A. Bartuska, I. A. Baste, A. Bilgin, E. Brondizio, K. M. A. Chan, V. E. Figueroa, A. Duraiappah, M. Fischer, R. Hill, T. Koetz, P. Leadley, P. Lyver, G. M. Mace, B. Martin-Lopez, M. Okumura, D. Pacheco, U. Pascual, E. S. Pérez, B. Reyers, E. Roth, O. Saito, R. J. Scholes, N. Sharma, H. Tallis, R. Thaman, R. Watson, T. Yahara, Z. A. Hamid, C. Akosim, Y. Al-Hafedh, R. Allahverdiyev, E. Amankwah, T. S. Asah, Z. Asfaw, G. Bartus, A. L. Brooks, J. Caillaux, G. Dalle, D. Darnaedi, A. Driver, G. Erpul, P. Escobar-Eyzaguirre, P. Failler, A. M. M. Fouda, B. Fu, H. Gundimeda, S. Hashimoto, F. Homer, S. Lavorel, G. Lichtenstein, W. A. Mala, W. Mandivenyi, P. Matczak, C. Mbizvo, M. Mehrdadi, J. P. Metzger, J. B. Mikissa, H. Moller, H. Mooney, P. Mumby, H. Nagendra, C. Nesshover, A. A. Oteng-Yeboah, G. Pataki, M. Roué, J. Rubis, M. Schultz, P. Smith, R. Sumaila, K. Takeuchi, S. Thomas, M. Verma, Y. Yeo-Chang, and D. Zlatanova. 2015. The IPBES Conceptual Framework - connecting nature and people. Curr Opin Environ Sustain 14:1-16.

Fisher, J.A., G. Patenaude, K. Giri, K. Lewis, P. Meir, P. Pinho, M. D. A. Rounsevell, and M. Williams. 2014. Understanding the relationships between ecosystem services and poverty alleviation: A conceptual framework. Ecosyst Serv 7:34-45. doi:10.1016/j.ecoser.2013.08.002.

L. A. Garibaldi, B. Gemmill-Herren, R. D'Annolfo, B. E. Graeub, S. A. Cunningham, and T. D. Breeze. 2017. Farming Approaches for Greater Biodiversity, Livelihoods, and Food Security. Trends Ecol Evol 32:68-80. doi:10.1016/ j.tree.2016.10.001.

Hevia, V., B. Martín-López, S. Palomo, M. García-Llorente, F. de Bello, and J. A. González. 2017. Trait-based approaches to analyze links between the drivers of change and ecosystem services: Synthesizing existing evidence and future challenges. Ecol Evol 7:831-844. doi:10.1002/ece3.2692.

Kremen, C., and A. Miles. 2012. Ecosystem services in biologically diversified versus conventional farming systems: benefits, externalities, and trade-offs. Ecol Soc 17.

Laterra, P., and L. Nahuelhual. 2015. Internalización de los servicios ecosistémicos en el ordenamiento territorial rural: bases conceptuales y metodológicas in Paruelo, J. M., E. G. Jobbágy, P. Laterra, M. Hernán Diéguez, A. García Collazo, and A. Panizza (eds.). Ordenamiento Territorial Rural. Conceptos, Métodos y Experiencias. FAO-MINAGRIFAUBA. Pp. 86-106.

Laurans, Y., and L. Mermet. 2014. Ecosystem services economic valuation, decision-support system or advocacy? Ecosyst Serv 7:98-105. doi:10.1016/j.ecoser.2013.10.002.

Laurans, Y., A. Rankovic, R. Billé, R. Pirard, and L. Mermet. 2013. Use of ecosystem services economic valuation for decision making: questioning a literature blindspot. J Environ Manage 119:208-219. doi:10.1016/j.jenvman.2013.01.008.

Martínez-Harms, M. J., and P. Balvanera. 2012. Methods for mapping ecosystem service supply: a review. Int J Biodivers Sci Ecosyst Serv Manag 8:17-25.

Martínez-Harms, M. J., B. A. Bryan, P. Balvanera, E. A. Law, J. R. Rhodes, H. P. Possingham, and K. A. Wilson. 2015. Making decisions for managing ecosystem services. Biol Conserv 184:229-238. doi:10.1016/j.biocon.2015.01.024.

Mastrangelo, M. E., F. Weyland, L. P. Herrera, S. H. Villarino, M. P. Barral, and A. D. Auer. 2015. Ecosystem services research in contrasting socio-ecological contexts of Argentina: Critical assessment and future directions. Ecosyst Serv 16:63-73. doi:10.1016/j.ecoser.2015.10.001.

MEA. 2005. Ecosystems and human well-being: Synthesis. Millennium Ecosystem Assessment. World Resources Institute. Washington, DC: Island Press.

Nahuelhual, L., P. Laterra, S. Villarino, M. Mastrangelo, A. Carmona, A. Jaramillo, P. Barral, and N. Burgos. 2015. Mapping of ecosystem services: Missing links between purposes and procedures. Ecosyst Serv (best practices for mapping ecosystem services) 13:162-172. doi:10.1016/j.ecoser.2015.03.005.

Olaya Rodríguez, M., M. Escobar Lizarazo, A. Cusva, C. Lasso Alcalá, M. Londoño Murcia. 2017. Mapeo del servicio ecosistémico de alimento asociado a la pesca en los humedales interiores de Colombia. Ecol Austral 27:123-133.

Pascual, U., P. Balvanera, S. Díaz, G. Pataki, E. Roth, M. Stenseke, R. T. Watson, E. Başak Dessane, M. Islar, E. Kelemen, V. Maris, M. Quaas, S. M. Subramanian, H. Wittmer, A. Adlan, S. Ahn, Y. S. Al-Hafedh, E. Amankwah, S. T. Asah, P. Berry, A. Bilgin, S. J. Breslow, C. Bullock, D. Cáceres, H. Daly-Hassen, E. Figueroa, C. D. Golden, E. GómezBaggethun, D. González-Jiménez, J. Houdet, H. Keune, R. Kumar, K. Ma, P. H. May, A. Mead, P. O'Farrell, R. Pandit, W. Pengue, R. Pichis-Madruga, F. Popa, S. Preston, D. Pacheco-Balanza, H. Saarikoski, B. B. Strassburg, M. van den Belt, M. Verma, F. Wickson, and N. Yagi. 2017. Valuing nature's contributions to people: the IPBES approach. Curr Opin Environ Sustain 26-27:7-16. doi:10.1016/j.cosust.2016.12.006. 
Posner, S. M., E. McKenzie, and T. H. Ricketts. 2016. Policy impacts of ecosystem services knowledge. Proc Natl Acad Sci 113:1760-1765.

Rey Benayas, J. M., M. P. Barral, and P. Meli. 2017. Lecciones de cuatro meta-análisis globales sobre la restauración de la biodiversidad y los servicios ecosistémicos. Ecol Austral 27:193-198.

Rubio, M.C., C. Rubio, M. Salomón and E. Abraham. 2017. Conservation of ecosystem services in high-altitude Andean wetlands: social participation in the creation of a natural protected area. Ecol Austral 27:177-192.

Rusch, V., G. Rusch, A. Goijman, S. Varela, and L. Claps. 2017. Ecosystem services to support environmental and socially sustainable decision-making. Ecol Austral 27:162-176.

Seppelt, R., C. F. Dormann, F. V. Eppink, S. Lautenbach, and S. Schmidt. 2011. A quantitative review of ecosystem service studies: approaches, shortcomings and the road ahead. J Appl Ecol 48(3):630-636. doi:10.1111/j.13652664.2010.01952.x.

Tallis, H., and S. Polasky. 2009. Mapping and valuing ecosystem services as an approach for conservation and naturalresource management. Ann N Y Acad Sci 1162:265-283.

Tengö, M., E. S. Brondizio, T. Elmqvist, P. Malmer, and M. Spierenburg. 2014. Connecting Diverse Knowledge Systems for Enhanced Ecosystem Governance: The Multiple Evidence Base Approach. AMBIO 43:579-591. doi:10.1007/s13280014-0501-3.

Teruya, J., L. Mastrantonio, and J. Portela. 2017. Evaluación Biofísica de Servicios Ecosistémicos en la Cuenca del Arroyo Grande, Tunuyán, Mendoza. Ecol Austral 27:113-122.

van Riper, C. J., A. C. Landon, S. Kidd, P. Bitterman, L. A. Fitzgerald, E. F. Granek, S. Ibarra, D. Iwaniec, C. M. Raymond, and D. Toledo. 2017. Incorporating sociocultural phenomena into ecosystem-service valuation: the importance of critical pluralism. BioScience 67:233-244.

Viglizzo, E. F., J. M. Paruelo, P. Laterra, and Jobbágy. 2012. Ecosystem service evaluation to support land-use policy. Agric Ecosyst Environ 154:78-84. doi:10.1016/j.agee.2011.07.007. 